\title{
Reevaluation of the Correlations between Ultrasound Features of Thyroid Nodules and Grades of Bethesda Classification
}

\author{
Ermal Tako ${ }^{1 *(D)}$, Blerina Cela ${ }^{1}$, Majlinda Ikonomi ${ }^{2}$ \\ ${ }^{1}$ Department of Radiology, American Hospital 2, Tirana, Albania; ${ }^{2}$ Department of Anatomical Pathology, National University \\ Hospital Mother Theresa, Hygeia Hospital, Tirana, Albania
}

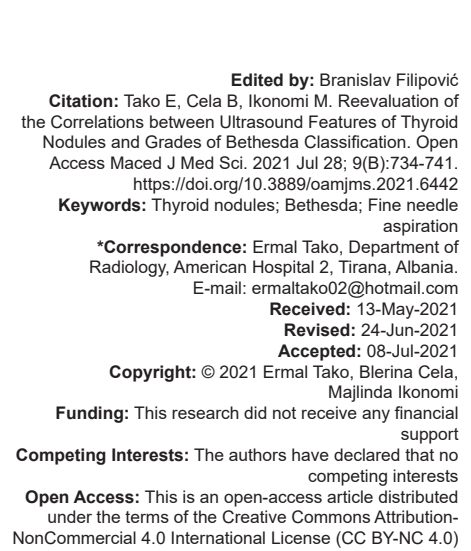

\section{Introduction}

The core of our study raises a question that is mentioned widely in medical opinions before: Which nodule could be malignant based on ultrasound (US) features and subsequently which of them need intervention. To answer these questions, we have to do a quick review of the imaging modalities which are used to examine thyroid nodules (THNs) and its US features.

The US has created a real revolution in the diagnosis of pathologies of the thyroid gland in general and THNs in particular. Unlike in the rest of the organs of the human body, the other imaging modalities have a limited role in thyroid examination. Despite the higher contrast resolution in magnetic resonance imaging and the higher spatial resolution with computed tomography scans (CT), the US remains the best modality for assessing THNs [1] (Figure 1). In scintigraphy (thyroid scan), the normal thyroid demonstrates uniform symmetrical uptake of radioactive material [2]. A THN is considered "hot" when it causes focal accumulation of radioactive material and "cold" when it gives a photopenic defect. It is valuable in tracking of a THN when TSH is low. In the case of a hot nodule with low TSH, the nodule malignancy is rare, so fine needle aspiration (FNAB) is not advised [3]. Despite the relatively high ability of scintigraphy to exclude benign nodules, the poor spatial 


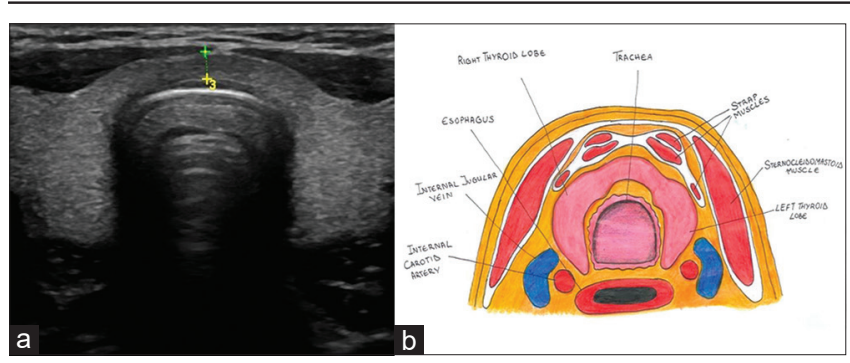

Figure 1: Ultrasound view of the normal thyroid gland (a). Schematic display of the anatomic structures in the cross-sectional view of the thyroid region (b)

resolution of this method makes it impossible to use it for FNAB orientation. Positron emission tomography (PET) in normal thyroid shows similar uptakes to adjacent skeletal muscle of 2-[fluorine-18] fluoro-2-deoxy-Dglucose (FDG). THNs are discovered randomly in 2-3\% of PET/CT examinations [4]. Both benign and malignant nodules show increased FDG uptake compared to normal adjacent parenchyma [5]. Although some studies show higher uptake in malignant nodules than in benign ones, there is still no SUV threshold in PET/CT to tell if a nodule is malignant or benign. For this reason, although nodules with high SUV are 14-40\% more likely to be malignant, further evaluation with US and FNAB is necessary [4]. Until now, the other modalities have minimal importance in the study of THNs. This is the reason why their US features remain still an area of research. In the recent years, elastography of thyroid gland has been a complementary examination in the determination of malignant nodules. With this method, due to the higher consistency, malignant nodules are distinguished from adjacent muscular tissue and benign nodules.

THN can be discovered in $4-8 \%$ of the population by palpation and in $40-50 \%$ of the population by US. Only $5 \%$ of THNs are malignant, of which, $75-80 \%$ are papillary carcinomas. Most part of THNs is hyperplastic benign nodules or adenomas [6]. THN and thyroid malignancies have a predilection for female gender, with a ratio of $4: 1$ and $2-3: 1$, respectively. In general, the likelihood of a malignant THN is higher in men and in patients under 15 and over 45 years of age [7]. Although many features of THN have been studied in terms of distinguishing whether they are malignant or benign, FNAB remains the main tool in THN management [6]. However, given the financial cost, potential complications, and anxiety of the patient, it is unjustified to puncture each THN. For this reason, various authors and associations have designed classifications in an attempt to determine which of the nodules should be punctured. Some of the most popular classifications are [8]: TI-RADS (Horvath et al. 2009); American College of Radiology (ACR) - TI-RADS; European (EU) - TI-RADS; Korean (K) - TI-RADS; AACE/ACE/AME; ATA classification and British Thyroid Association US classification. Despite these efforts, none of the above classifications have found a wide practical use, but according to a comparative study by Grani et al., the ACR-TI-RADS classification was found to be most effective in reducing the number of cytologies (at 268 out of 502), with the lowest false negativity of $2.2 \%$ (NPV, 97.8\%; 95\% Cl: 95.2-99.2\%), with a sensitivity of $83 \%$, specificity $56.2 \%$ and PPV $12.8 \%$ [9]. That is why THNs included in the study are classified according to ACR-TI-RADS. To understand the US features of THN, it is better first to know normal thyroid glands US appearance and what are THNs. The normal aspect of thyroid gland in the US is hyperechoic, homogeneous and with fine echostructure (Figure 1) [10]. The approximate size of the lobes in adults is 4-6 cm in craniocaudal diameter and 1.3-1.8 cm in anteroposterior and transverse diameters. The isthmus is $3 \mathrm{~mm}$ in anteroposterior diameter [11]. THN is defined by the American Thyroid Association "as a discrete lesion within the thyroid gland which is radiologically distinct from the surrounding thyroid parenchyma [12] (Figure 2). Below, it is a concentrated ACR-TI-RADS classification (Tables 1 and 2). This classification applies only to nodules and not to normal thyroid [13]. In 2007 the Bethesda classification classified the histopathologic findings of the aspirated material into 6 categories [14]. To clearly understand the Bethesda classification, one must know the histopathology of THN and diffuse pathologies of thyroid gland presented briefly below:
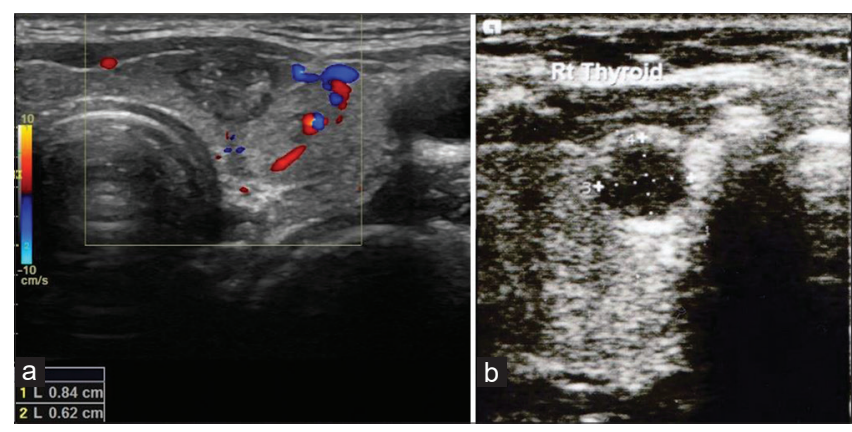

Figure 2: ( $a$ and b) Hyperechogenic, heterogenic, nodus, surrounded by a hypoechogenic halo, $8 \mathrm{~mm}$ in size, with microcalcifications and without vascularization inside, subcapsular, in the anterior contour of the left thyroid lobe, classified Bethesda V in cytology. As can be seen the nodus is sonographically distinct from surrounding parenchyma (a). Marked hypoechogenic nodus with irregular borders, $6 \mathrm{~mm}$ in size, classified Bethesda V (b)

\section{Benign lesions}

- Benign follicular nodules (Figure 3)

- Adenomatoid nodules

- Follicular adenoma (Figure 4)

- Hurthle cell adenoma

- De Quervain thyroiditis

- Chronic lymphocytic thyroiditis hashimoto (Figure 5)

- Malignant lesions

- Papillary carcinoma (Figure 6)

- Follicular carcinoma (Figure 7)

- Hurthle cell carcinoma

- Poorly differentiated carcinoma

- Poorly differentiated anaplastic carcinoma (Figure 8) 
Table 1: ACR-TI-RADS. Points according to ultrasonic features

\begin{tabular}{|c|c|c|c|c|}
\hline Consistency & Echogenicity & Shape & Contours & Classification \\
\hline Cystic 0 point & Anechogenic 0 point & Widder than taller 0 point & Clear 0 point & Without calcifications 0 point \\
\hline Spongiform 0 pike & Hyperechogenic or isoechogenic 1 point & Taller than Widder 3 points & Unclear 0 point & Comet-tail calcifications 0 pike \\
\hline Solidocystic 1 point & Hypoechogenic 2 point & & Irregular lobular 2 points & Macrocalcifications 1 point \\
\hline Solid or almost solid 2 points & Marked hypoechogenic 3 point & & Extralobar protrusion 3 points & $\begin{array}{l}\text { Peripheric or rime calcifications } 2 \text { points } \\
\text { Microcalcifications } 3 \text { points }\end{array}$ \\
\hline
\end{tabular}

Table 2: ACR-TI-RADS. Categories with respective recommendations

\begin{tabular}{lll}
\hline Points & Classification & Recommendations \\
\hline TR1: 0 point & Unsuspicious benign $0.3 \%$ & No FNA required \\
TR2: 2 points & Unsuspicious $1.5 \%$ & No FNA required \\
TR3: 3 points & Mildly suspicious $4.8 \%$ & $\geq 1.5 \mathrm{~cm}$ follow-up, $\geq 2.5 \mathrm{~cm}$ FNA \\
& & follow-up: 1,3, and 5 years \\
TR4: $4-6$ points & Suspicious $9.1 \%$ & $\geq 1.0 \mathrm{~cm}$ follow-up, $\geq 1.5 \mathrm{~cm}$ FNA \\
& & Follow-up: $1,2,3$, and 5 years \\
TR5: $\geq 7$ points & Highly suspicious $35 \%$ & $\geq 0.5 \mathrm{~cm}$ follow-up, $\geq 1.0 \mathrm{~cm}$ FNA \\
& & annual follow-up for up to 5 years \\
\hline
\end{tabular}
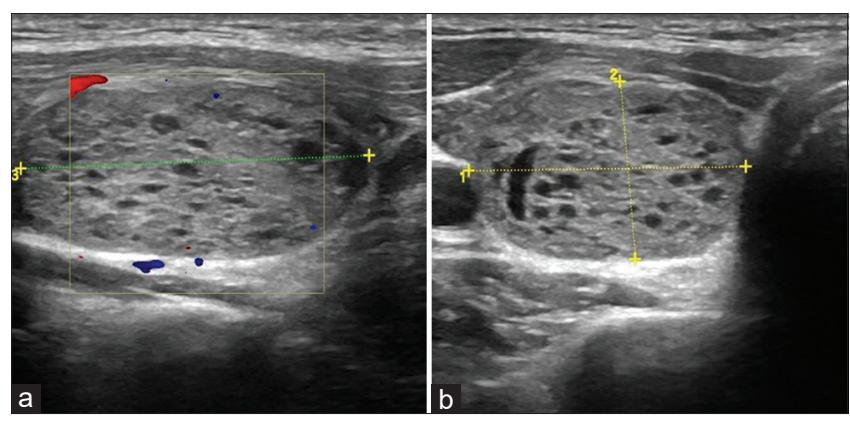

Figure 3: Solitary, spongiform, nodus $25 \times 16 \mathrm{~mm}$ in size, without vacsularization inside, extralobar, in the lower pole of the right thyroid lobe, classified Bethesda 3. In the post-operatory biopsy resulted benign follicular nodus

- Medullary carcinoma

- Lymphoma

- Metastasis

Because the real reason of this study is the differentiation of malignant pathologies, it is important to take a brief look at some of the most important categories.

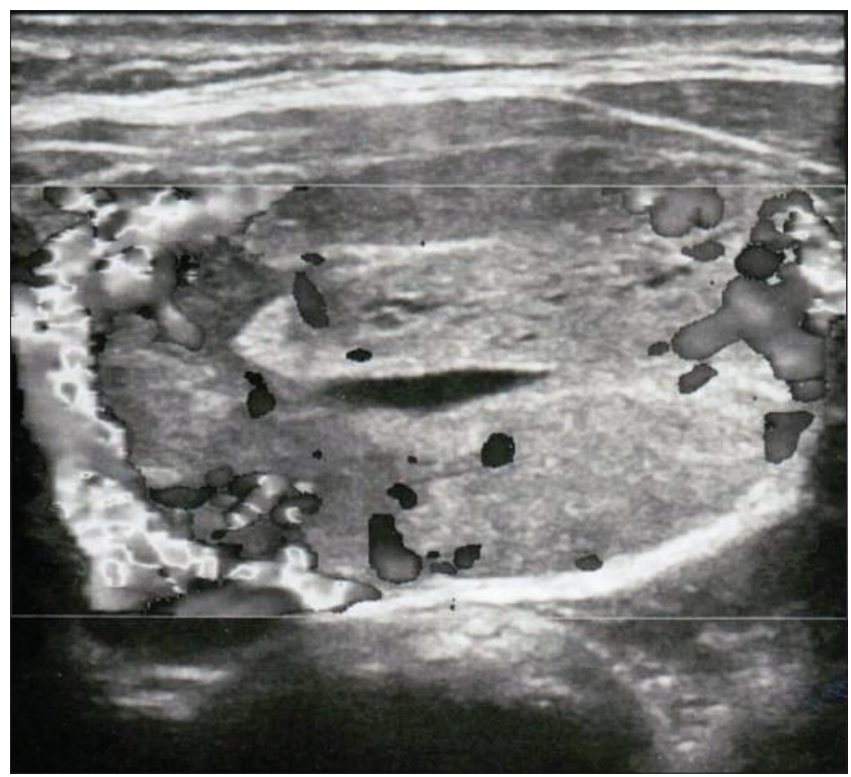

Figure 4: Hyperechogenic nodus with cystic degeneration inside and peripheral vascularization of the left thyroid lobe, classified Bethesda $V$. In the post operatory biopsy resulted microfollicular adenoma

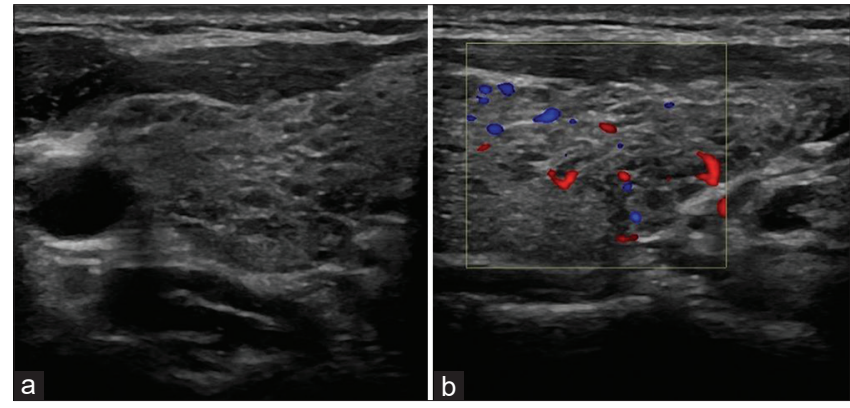

Figure 5: ( $a$ and b) Thyroiditis Hashimoto. Right lobe, transverse view with "leopard skin" pattern (a). Longitudinal view with moderate vascularization (b)

- Papillary carcinoma, which as mentioned above comprised the most part of malignant pathologies of thyroid gland, has a papillary architecture composed of follicular cells with distinct nuclear features [15]. When the FNAB is positive for papillary carcinomas, total thyroidectomy is indicated, because of its multifocal nature, with or without subsequent ablation with radioactive iodine. If FNAB is suspicious, lobectomy with "intraoperative frozen biopsy" is recommended for confirmation. If the result is positive, the other lobe is removed. The prognosis of papillary carcinoma is generally good (10 years relative survival at 93\%). Bad prognostic factors are age over 45 years and advanced stage of the tumor. Total body scan with 131 I is useful in assessing disease recurrence after thyroidectomy and ablation [7].
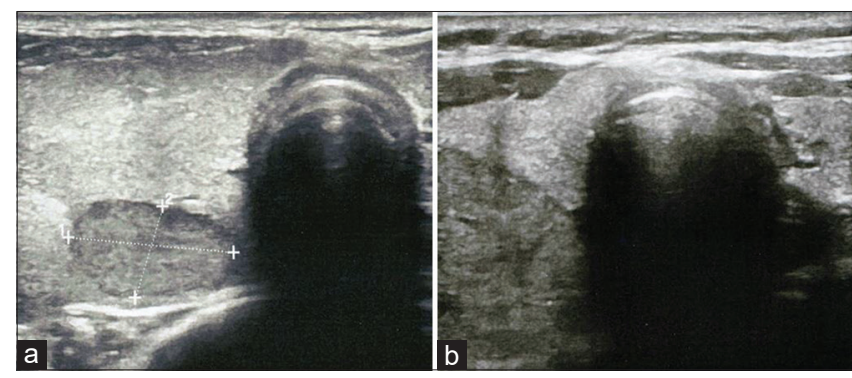

Figure 6: ( $a$ and b) Solitary, hypoechogenic nodus, with clear contours, of the right thyroid lobe, classified Bethesda V. In the post operatory biopsy resulted papillary carcinoma, follicular variant (a). Needle shadow during the biopsy (b)

Follicular carcinoma consists of follicular cells with capsular or vascular invasion [16] and comprises $11 \%$ of thyroid malignancies [7]. Both, follicular adenoma and carcinoma, in FNAB are considered as follicular neoplasms or suspected as such and are reported in 

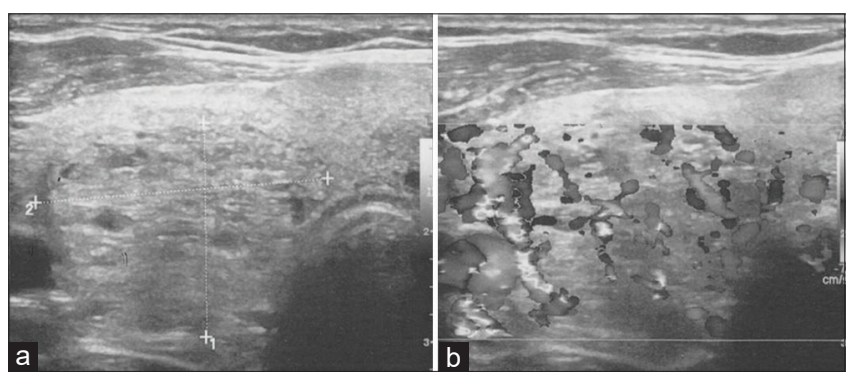

Figure 7: (a and b) Solitary, heterogenic, mainly hyperechogenic nodus, with clear contours, of the right thyroid lobe, classified Bethesda IV. In the post operatory biopsy resulted follicular carcinoma (a). Central vascularization (b)

$6-12 \%$ of FNABs [17], $15-30 \%$ of which are malignant [16]. It is more common in men, the elderly, and those with large nodules [16]. Treatment modalities include total thyroidectomy with or without ablation with radioactive iodine for the invasive form and lobectomy or isthmus removal for minimally invasive forms. The prognosis is generally good with 10 years of relative survival in $85 \%$ of cases. Poor prognostic factors are older age over 45 years and advanced stage [18].

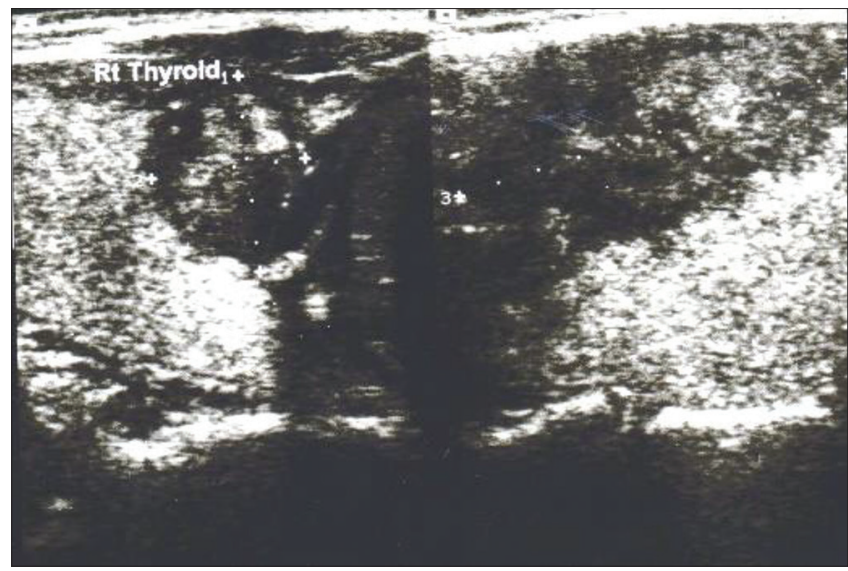

Figure 8: Poorly differentiated anaplastic carcinoma of the right thyroid lobe, transverse and longitudinal view. In the superior medial side of the right lobe, it is seen a heterogenic, mostly hypoechoic, solid, infiltrative mass with unclear contours. In the post operatory biopsy resulted anaplastic carcinoma

- $\quad$ Anaplastic (or undifferentiated) carcinoma is a highly malignant neoplasm with the features of a high-grade carcinoma. It comprises $2 \%$ of primary thyroid malignancies [16]. It has a poor prognosis with 5 months to 1-year survival in only $20 \%$ of cases [19]. Surgical removal is not always an option due to its early local spread. If it can be performed, it is necessary to be done immediately and by a surgeon specialized in surgeries for thyroid pathologies of this kind, because reintervention is often impossible. First of all, accurate staging plays a crucial role. Then, chemotherapy and radiotherapy can be applied. Anaplastic carcinoma does not uptake iodine so the diagnosis with thyroid scan and ablation with radioactive iodine cannot be applied [15].
Medullary carcinoma originates from neuroendocrine $\mathrm{C}$ cells of thyroid, which secrete calcitonin [20] and accounts for $4 \%$ of all primary thyroid malignancies [7]. Even this pathology does not uptake iodine therefore, similarly to anaplastic carcinoma, thyroid scan diagnosis and radioactive iodine therapy are not an option. About $80 \%$ of cases are sporadic and only $20 \%$ have a familial character, with type $2 a$ of multiple endocrine neoplasia the most common familial condition [7]. The primary treatment is total thyroidectomy [16]. The prognosis is intermediate with $75 \%$ 10 years of relative survival [7].

Established in 2007, the Bethesda System for Thyroid Cytology Reports is a classification with six categories that comprise the explanations of risks for malignancy and recommendations for further management [14] (Table 3).

\section{Aim of the study}

The evaluation of the statistical correlation between US features of THN and grades of Bethesda classification, to correctly select the patients who must undergo FNAB.

\section{Material and Methods}

In this study, we have included 260 cytologies of thyroid gland during a period from 2014 to 2018 . The procedures are performed at the radiology department of Hygeia Hospital with GE Logiq P7 (GE Healthcare, Chicago, Illinois, USA), GE Logiq E9 (Chicago, Illinois, USA) and SIEMENS ACUSON NX2 (Siemens Healthcare $\mathrm{GmbH}$, Erlangen, Germany) US machines. In our study are excluded the cases with a high risk of hemorrhage and the patients which did not accept the anesthetic procedure because of anxiety. Table 4 is displayed US features of THNs combined with their respective Bethesda classification and in Table 5, THNs according to ACR/TI RADS categories combined with Bethesda classification.

The technique that has been used is the same as the one that is described in the literature [15]. The patient is placed on the examination table in the supine position with the neck extended, which optimizes visualization of the thyroid during the US examination. The radiologist usually stands near the patient's chest, which we consider to be the most anatomically intuitive approach; in some cases, however, the radiologist may need to stand near the patient's head. Preliminary US of the area of interest with a high-resolution 10-12$\mathrm{MHz}$ linear-array transducer is performed to determine a suitable approach to the nodule. The patient is asked 
Table 3: The Bethesda classification (Figures 9 and10) [21]

\begin{tabular}{|c|c|c|c|}
\hline Data for every category categories & Content & Risk for malignancy & Recommendations \\
\hline I. Nondiagnostic or unsatisfactory & Not enough cells for diagnosis or with high hemorrhagic content ${ }^{\star}$ & - & $\begin{array}{l}\text { Recommended repeating the examination after } \\
\text { some weeks }\end{array}$ \\
\hline II. Benign & Enough cells to define the nodule as benign & $0-3 \%$ & Recommended careful clinical follow-up \\
\hline $\begin{array}{l}\text { III. Atypia with undefinite significance or } \\
\text { follicular nodule with undefinite significance }\end{array}$ & $\begin{array}{l}\text { Enough cells, but is not possible to definite if it is a benign or } \\
\text { malignant process }\end{array}$ & $5-15 \%$ & Redo the examination after 6 weeks \\
\hline $\begin{array}{l}\text { IV. Follicular neoplasm or suspicion for follicular } \\
\text { neoplasm }\end{array}$ & $\begin{array}{l}\text { Follicular tumor or adenoma, but in cannot be defined in } \\
\text { cytology }\end{array}$ & $15-30 \%$ & Recommended lobectomy \\
\hline V. Suspicious for malignancy & $\begin{array}{l}\text { The nodules are suspicious for malignancy, but in cytology } \\
\text { cannot be defined the type of tumor }\end{array}$ & $60-75 \%$ & Recommended total thyroidectomy \\
\hline VI. Malignant & The malignancy of the nodule is certain & $97-99 \%$ & Total thyroidectomy \\
\hline
\end{tabular}

${ }^{*}$ Category I happens when a cyst is punctated or when a large amount of blood is aspirated.

not to swallow or speak during FNAB, which helps limit thyroid movement. The neck is then cleansed with chlorhexidine-alcohol or povidone-iodine, which is allowed to dry. Sterile towels are placed around the procedural field, and a sterile cover is placed over the US probe. The probe is positioned for optimal visualization of the target nodule.

Table 4: Ultrasound features of THN with respective Bethesda categories

\begin{tabular}{llllllll} 
Ultrasound features of THN & \multicolumn{3}{l}{$\begin{array}{l}\text { Categories of Bethesda } \\
\text { classification }\end{array}$} & \multicolumn{3}{l}{ Nr. for every feature } \\
\cline { 2 - 7 } & I & II & III & IV & V & VI & \\
\hline Hyperechogenic & 15 & 42 & 44 & 21 & 12 & 1 & 135 \\
Hypoechogenic & 5 & 13 & 16 & 14 & 7 & 0 & 55 \\
Mixed & 0 & 10 & 4 & 1 & 1 & 1 & 17 \\
solid & 20 & 65 & 64 & 36 & 20 & 2 & 207 \\
Solido-cystic & 1 & 16 & 14 & 4 & 0 & 0 & 35 \\
cystic(anechogenic) & 1 & 8 & 0 & 0 & 0 & 0 & 9 \\
Sponge & 2 & 3 & 2 & 1 & 1 & 0 & 9 \\
Microcalcifications & 1 & 17 & 23 & 8 & 8 & 0 & 57 \\
Macrocalcifications & 0 & 8 & 2 & 2 & 0 & 0 & 12 \\
taller than wide & 0 & 2 & 1 & 2 & 0 & 0 & 5 \\
Extralob. protr.* & 2 & 6 & 9 & 9 & 8 & 0 & 34 \\
Central vasc.** & 7 & 22 & 9 & 18 & 4 & 1 & 61 \\
Peripheric vasc. & 17 & 63 & 70 & 28 & 20 & 1 & 199 \\
Regular contours & 19 & 87 & 67 & 36 & 19 & 2 & 230 \\
Irregular contours & 3 & 8 & 12 & 4 & 3 & 0 & 30 \\
Females & 18 & 90 & 77 & 38 & 19 & 1 & 243 \\
Males & 5 & 0 & 5 & 1 & 5 & 1 & 17 \\
Solitary & 16 & 57 & 42 & 26 & 14 & 2 & 157 \\
Multinodular struma & 6 & 37 & 38 & 15 & 7 & 0 & 103 \\
Right lobe & 13 & 48 & 39 & 20 & 13 & 1 & 134 \\
Left lobe & 10 & 42 & 40 & 20 & 7 & 1 & 120 \\
isthmus & 0 & 3 & 0 & 1 & 2 & 0 & 6 \\
Total number of THN & & & & & & & 260 \\
\hline
\end{tabular}

THN: Thyroid nodule. *Extralobar protrusion. ${ }^{*}$ Central vascularisation

About 5-10 mL of $1 \%$ lidocaine hydrochloride solution is infiltrated into the skin and subcutaneous tissues with a 25-gauge needle for local anesthesia. FNAB is subsequently performed under continuous US guidance, with the needle oriented either paralle (Figure 9) or perpendicular to the US probe. FNAB is performed using 27-gauge needles. A total of six passes are made for each nodule selected. First, three passes are made without suction using the capillary technique. Three more passes are then made with continuous $0.5-1-\mathrm{mL}$ suction applied to an attached $10-\mathrm{mL}$ syringe using the aspiration technique.

Table 5: THN according to ACR/TI RADS and Bethesda categories

\begin{tabular}{llllllll}
\hline Categories & BI & BII & BIII & BIV & BV & BVI & Nr. \\
\hline TR1 & 3 & 20 & 9 & 4 & 0 & 0 & 36 \\
TR2 & 2 & 11 & 5 & 1 & 2 & 0 & 21 \\
TR3 & 11 & 28 & 27 & 14 & 5 & 2 & 87 \\
TR4 & 4 & 29 & 25 & 10 & 8 & 0 & 76 \\
TR5 & 3 & 7 & 12 & 11 & 7 & 0 & 40 \\
Nr. & 23 & 95 & 78 & 40 & 22 & 2 & 260 \\
& 196 & & & 64 & & & \\
\hline
\end{tabular}

Each pass consists of approximately 50 vigorous controlled excursions of the needle through the nodule over a 20 -s period. For solid lesions, multiple peripheral regions should be sampled to increase the adequacy rate. For mixed cystic and solid lesions or predominantly cystic lesions with a solid mural nodule, the solid component of the lesion is targeted to improve diagnostic yield. If color Doppler analysis shows relative increased vascularity in a portion of a nodule, this region is similarly targeted. For vascular nodules, the small (27-gauge) needle size, capillary technique, and short needle dwell time are particularly important for improving diagnostic yield by decreasing the amount of blood in the aspirate. If a prior biopsy specimen was reported as nondiagnostic or indeterminate, we perform combined FNAB and core biopsy. Upon completion of biopsy, gentle manual pressure may be applied, the skin is cleansed, and a sterile dressing is put in place. The patient is discharged immediately after the procedure. No major complications requiring intervention or hospitalization have been reported. Most of cases experience a slight pain and discomfort. In rare cases, the puncture may cause a small subcapsular or intranodal hematoma that resolves spontaneously (Figure 10).

Firstusingtheztest, wecompared thepercentage occupied by the Bethesda categories that are indicative of surgery (BIV + BV + BVI) at US features that suspect malignancy (hypoechogenicity, microcalcifications, abnormal contours, central vascularization), with the

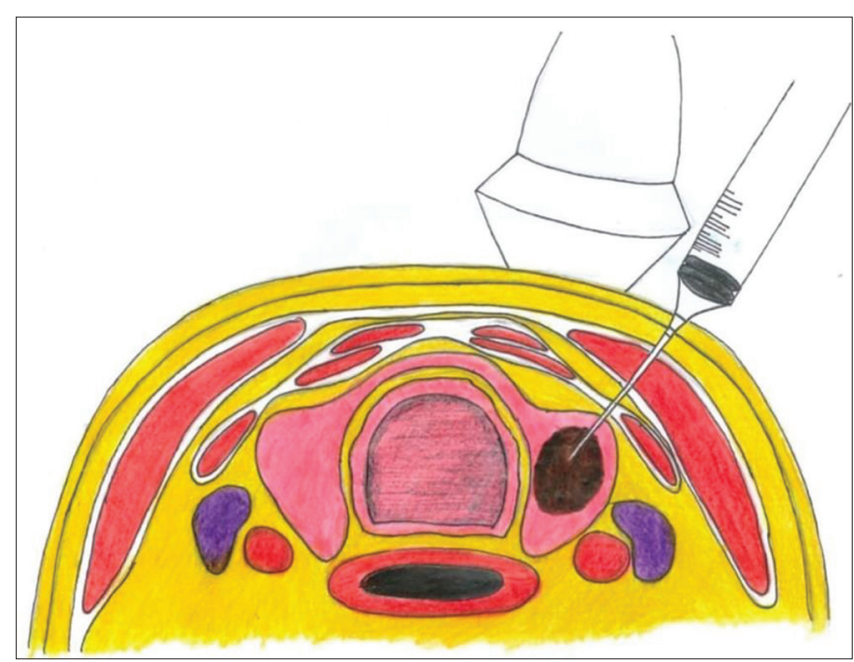

Figure 9: The schematic display of the needle pathway during a thyroid nodule punction 


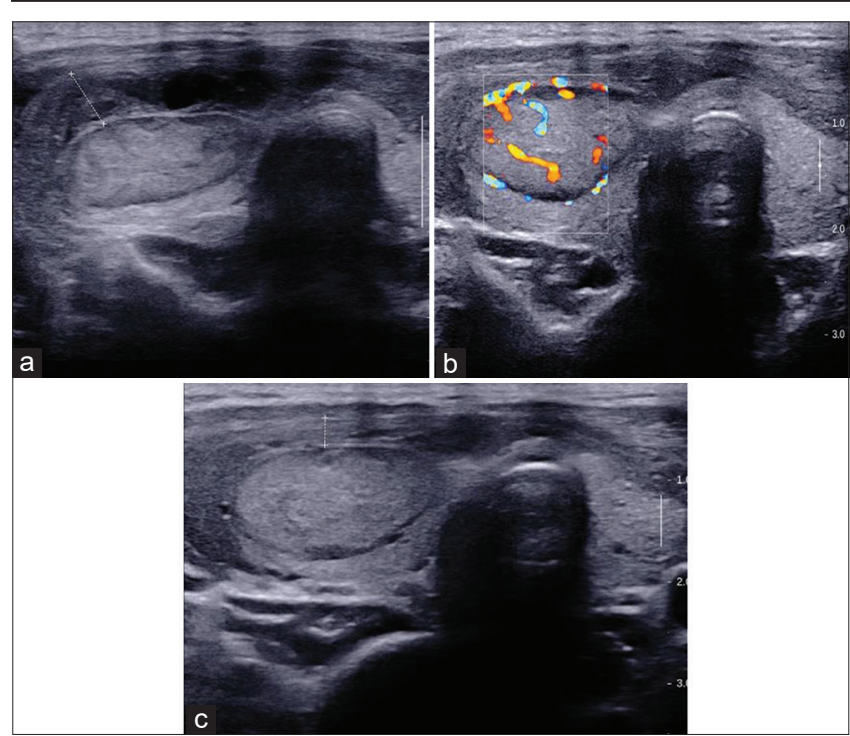

Figure 10: (a and c) Subcapsular hematoma immediately after the punction of an anterior, hyperechoic, well vascularized solitary nodus of the right lobe (Bethesda IV) (a). Perinodal and central vascularization (b). After $1 \mathrm{~h}$ treatment with compression and ice on the left lobe, almost the half of the hematoma is absorbed (c)

percentage occupied by group (BIV $+\mathrm{BV}+\mathrm{BVI}$ ) at the US features which indicate benignity (hyperechoic, no microcalcifications, peripheral vascularization, cysticsolidocystic, spongiform, normal contours).

Furthermore, we have evaluated utilizing the odds ratio if there was a correlation between TR4 and TR5 categories in ACR/TIRADS classification and the categories (BIV+BV+BVI) for any statistical significance. The significance of the dimensions of the nodule was tested as an indicator for surgical intervention. For this purpose, the percentage occupied by the nodules with a diameter larger than $1.5 \mathrm{~cm}$ at BIV + BV + BVI group was compared with the percentage occupied by nodules smaller than $1.5 \mathrm{~cm}$ at BIV + BV + BVI. In addition, we observed if there was a strong statistical connection between nodules larger than $1.5 \mathrm{~cm}$ and the Bethesda categories that suggested malignancy. There was no statistical test made for the features "taller than wide" and microcalcifications because of the small number of cases. It was also made a comparison of percentages $(B I V+B V+B V I)$ even for three clinical features: men versus women, solitary nodule versus multinodular goiter, left lobe versus right lobe. We compared the percentages occupied by the (BIV + BV + BVI) group of categories in patients over 45 years old with the percentages occupied by this group at patients younger than 45-years-old. We also noted which of Bethesda categories is more frequent.

\section{Results}

After performing statistical tests, it was found that, for the features, hypoechoic $\left(P_{b}=38.18 \%\right)$ versus hyperechoic $\left(P_{a}=24.34 \%\right)$, according to the $z$ test $(p<0.05)$, the difference between the percentages was statistically significant $(z=-1,958 ; p=0.0025<0.05)$. In the case of microcalcifications $\left(P_{b}=28.07 \%\right)$ versus without microcalcifications $\left(P_{a}=23.65 \%\right)$, according to the $z$-score test $(p<0.05)$, the change was insignificant $(z=-0.685 ; p=0.2467>0.05)$. For the feature of central vascularization $\left(P_{b}=37.7 \%\right)$ versus peripheral vascularization $(\mathrm{Pa}=24.75 \%)$, the comparison was made with the statistical test $z$-score according to which the difference is significant $(z=-1.98 ; p=0.0239<0.05)$. For the features of regular contours $\left(P_{a}=24.78 \%\right)$ versus irregular contours $\left(P_{b}=23.33 \%\right)$, the comparison was made with the statistical test $z$-score $(p<0.05)$ according to which the difference is insignificant $(z=$ $0.173 ; p=0.4313>0.05)$. For the position of the nodule in the lobe, extralobar nodule $\left(P_{b}=50 \%\right)$ versus intralobar nodule $\left(P_{a}=20.8 \%\right)$, the comparison was made with the statistical test $z$-score $(p<0.05)$ according to which the difference is significant $(z=-3,686 ; p=0.0001<0.05)$. For the group of features (solidocystic + cystic + spongiform) $\left(P_{a}=15.49 \%\right)$ versus solid $\left(P_{b}=28.57 \%\right)$, the comparison was made with the statistical test z-score $(p<0.05)$ according to which the difference is significant $(z=-2.17 ; p=0.015<0.05)$. For two clinical features mentioned above, the results were as follows: the comparison, female $\left(P_{a}=23.82 \%\right)$ versus male $\left(P_{b}\right.$ $=41.18 \%$ ), was made with the statistical test $z$-score $(p$ $<0.05$ ) according to which the difference is insignificant $(z=-1,593 ; p=0.0556>0.05)$. The comparison of multinodular $\left(P_{a}=21.36 \%\right)$ versus solitary $\left(P_{b}=\right.$ $26.75 \%$ ) nodules was made with the statistical test z-score $(p<0.05)$ according to which the difference is insignificant $(z=-0.987 ; p=0.1618>0.05)$. The comparison of left lobe $\left(P_{a}=23.33 \%\right)$ versus the right lobe $P_{b}=(25.37 \%)$ was made with the statistical test $z$-score $(p<0.05)$ according to which nodules with US features of malignancy have no lobar preferences $(z=-0.378$; $p$ $=0.3527>0.05 ; p=0.7054>0.05)$. Regarding the age, it was seen that the percentage of cases belonging to the categories $(\mathrm{BIV}+\mathrm{BV}+\mathrm{BVI})$ over 45 years old is higher than the percentage of cases (BIV + BV + BVI) under 45 years old and this difference according to the $z$-score test $(p<0.05)$ is statistically significant $(z$-score $=-2,828 ; p=0.0023$ ). However, based in the odds ratio, being over 45 years old is related weakly statistically to the categories (BIV + BV + BVI).

Regarding the size of the nodules, the comparison of percentages according to the z-score test $(p<0.05)$ showed that nodules larger than $1.5 \mathrm{~cm}$ occupy a percentage (59.4\%) statistically higher in the categories that suggest malignancy than the nodules below $1.5 \mathrm{~cm}(40.6 \%)(z=-2.121 ; p=0.017<0.05)$. The percentage of nodules larger than $1.5 \mathrm{~cm}$ in the categories that suggest for benign nature (which do not suggest surgery) is $60.2 \%$, almost the same as the percentage in the categories that suggest malignancy.

Based in the odds ratio ( $p<0.05)$, no relationship statistically significant was observed between the TR4 
category of the ACR/TI RADS classification and the (BIV $+\mathrm{BV}+\mathrm{BVI}$ ) categories of the Bethesda classification (odd $=0.931034 ; p=0.411384 ; z=0.223986)$. The relationship between the TR5 category and the (BIV + BV + BVI) categories of the Bethesda classification was statistically strong (odd = 3.094862; $p=0.000812 ; z=3.151623$ ).

The group of categories $(\mathrm{BI}+\mathrm{BII})$ occupies $37.7 \%$ of cases, categories (BIV + BV + BVI) occupy $31.5 \%$, while that BIII occupies $30.7 \%$ of cases. As can be seen, the differences between them are not significant, but it is impressive that only category BIII occupies approximately $1 / 3$ of all cases.

\section{Discussions}

Two of the most important US features of nodules that indicate for FNA are "hypoechogenicity" and extralobar location of THN, which is consistent with the results of almost all the other studies in this field. An important feature is the central vascularization of THN. That is why we recommend that it should be included in the ACR/TI RADS classification as well as in other similar classifications that do not contain it. Another strong feature is the consistency of THN. A solid nodus is more indicative than solidocystic or spongiform one. Age over 45 years old is also an indicative feature, but not of particular importance. The size of the nodule over $1.5 \mathrm{~cm}$ is an indicative feature, but not very important since it is found in almost the same percentage in the group of categories $(\mathrm{BI}+\mathrm{BII}+\mathrm{BIII})$.

Microcalcifications did not prove to be an important feature in the recommendation for FNA. This can have several reasons, as follows: (1) The evaluation was not done in real time but in retrospective based on footage and prints. (2) The examiner's experience in evaluating microcalcifications. (3) The type of machine and preset used for thyroid examination. (4) The number of cases probably should have been larger to make a more realistic assessment. The contours did not turn out to be a significant discriminatory feature, probably due to the limited number of cases. Clinical features such as gender, multinodular goiter or solitary nodule, being in the right or left lobe did not prove important to indicate a recommendation for FNA. It is worth noting here that for the feature men versus women, it is needed a separate study, with a larger number of cases, to draw a more realistic statistical conclusion.

\section{Conclusions}

The features that are more indicative for FNAB are hypoechogenicity, consistency, intranodal vascularization, and extralobar positioning. If a THN has one of the above features and has a dimension of more than $10 \mathrm{~mm}$, it has an indication for FNAB. The more of the above features a THN has the more indication for a FNAB it will have. The combination of US features that suggest malignancy, TR4, and TR5, with BIII category is a strong indicator for surgical intervention. The results of this study are similar with the results of prior studies and we could not distinguish any specific US feature that has an absolute indication for FNAB. The appropriate determination of the US features of a THN in correlation with the patient's clinic information will determine the proper indication for a FNAB.

\section{References}

1. Lew JI, Solorzano CC. Use of ultrasound in the management of thyroid cancer. Oncologist. 2010;15(3):253-8.

PMid:20215358

2. Sandler MP, Patton JA, McCook BM. Multimodality imaging of the thyroid gland. Baillieres Clin Endocrinol Metab. 1989;3(1):89-119.

PMid:2679526

3. Gharib H, Papini E, Paschke R, Duick DS, Valcavi R, Hegedüs L, et al. American association of clinical endocrinologists, associazione medici endocrinologi, and European thyroid association medical guidelines for clinical practice for the diagnosis and management of thyroid nodules: Executive summary of recommendations. Endocr Pract. 2010;16(3):468-75. https://doi.org/10.1007/bf03346587 PMid:20551008

4. Langer JE, Baloch ZW, McGrath C, Loevner LA, Mandel SJ. Thyroid nodule fine-needle aspiration. Semin Ultrasound CT MR. 2012;33(2):158-65. https://doi.org/10.1053/j.sult.2011.12.002

5. Intenzo CM, Dam HQ, Manzone TA, Kim SM. Imaging of the thyroid in benign and malignant disease. Semin Nucl Med. 2012;42(1):49-61. https://doi.org/10.1053/j. semnuclmed.2011.07.004

PMid:22117813

6. Desser TS, Kamaya A. Ultrasound of thyroid nodules. Neuroimaging Clin North Am. 2008;18(3):463-78. https://doi. org/10.1016/j.nic.2008.03.005

7. Tuttle RM, Ball DW, Byrd D, Dickson P, Duh QY, Ehya H, et al. National Comprehensive Cancer Network Clinical Practice Guidelines in Oncology: Thyroid Carcinoma, Version 2. New York: NCCN; 2013.

8. Horvath E, Silva CF, Majlis S, Rodriguez I, Skoknic V, Castro A, Rojas H, Niedmann JP, Madrid A, Capdeville F, Whittle C, Rossi $R$, Domínguez M, Tala $H$. Prospective validation of the ultrasound based TIRADS (Thyroid Imaging Reporting And Data System) classification: results in surgically resected thyroid nodules. Eur Radiol. 2017 Jun;27(6):2619-2628. doi: 10.1007/ s00330-016-4605-y.

PMid:27718080

9. Grani G, Lamartina L, Ascoli V, Bosco D, Biffoni M, Giacomelli L, et al. Reducing the number of unnecessary thyroid biopsies while improving diagnostic accuracy: Toward the "Right" TIRADS. J Clin Endocrinol Metab. 2019;104(1):95-102. https:// doi.org/10.1210/jc.2018-01674 


\section{PMid:30299457}

10. Loevner LA. Imaging of the thyroid gland. Semin Ultrasound CT MR. 1996;17(6):539-62. https://doi.org/10.1016/ s0887-2171(96)90003-7

11. Middleton WD, Kurtz AB, Hertzberg BS. Neck and chest. In: Thrall $\mathrm{JH}$, editor. Ultrasound: The Requisites. $2^{\text {nd }}$ ed. St Louis, Mo: Mosby; 2004. p. 244-77. https://doi.org/10.1016/ b978-0-323-01702-2.50016-5

12. American Thyroid Association (ATA) Guidelines Taskforce on Thyroid Nodules and Differentiated Thyroid Cancer, Cooper DS, Doherty GM, Haugen BR, Kloos RT, Lee SL, Mandel SJ, et al. Revised American thyroid association management guidelines for patients with thyroid nodules and differentiated thyroid cancer. Thyroid 2009;19(11):1167-214. https://doi.org/10.1089/ thy.2009.0110

PMid:19860577

13. Knipe H, Smith D. ACR Thyroid Imaging Reporting and Data System (ACR TI-RADS). Australia: Radiopedia; 2021.

14. Cibas ES, Ali SZ, NCl Thyroid FNA State of the Science Conference. The Bethesda system for reporting thyroid cytopathology. Am J Clin Pathol. 2009;132(5):658-65 https://doi. org/10.1309/ajcpphlwmi3jv4la

\section{PMid:19846805}

15. Nachiappan AC, Metwalli ZA, Hailey BS, Patel RA, Ostrowski ML, Wynne DM. The thyroid: Review of imaging features and biopsy techniques with radiologic-pathologic correlation. Radiographics. 2014;34(2):276-93. https://doi. org/10.1148/rg.342135067

PMid:24617678
16. Ali SZ, Cibas ES. The Bethesda system for reporting thyroid cytopathology. New York: Springer; 2009. p. 1-165.

17. Yang J, Schnadig V, Logrono R, Wasserman PG. Fine-needle aspiration of thyroid nodules: A study of 4703 patients with histologic and clinical correlations. Cancer. 2007;111(5):306-15. https://doi.org/10.1002/cncr.22955

PMid: 17680588

18. Ito $\mathrm{Y}$, Hirokawa M, Higashiyama $\mathrm{T}$, Takamura $\mathrm{Y}$, Miya $\mathrm{A}$, Kobayashi K, et al. Prognosis and prognostic factors of follicular carcinoma in Japan: Importance of postoperative pathological examination. World J Surg. 2007;31(7):1417-24. https://doi. org/10.1007/s00268-007-9095-2

PMid: 17534542

19. Agrawal S, Rao RS, Parikh DM, Parikh HK, Borges AM, Sampat MB. Histologic trends in thyroid cancer 1969-1993: A clinico-pathologic analysis of the relative proportion of anaplastic carcinoma of the thyroid. J Surg Oncol. 1996;63(4):251-5. https://doi.org/10.1002/ (sici)1096-9098(199612)63:4<251:aid-jso7>3.0.co;2-b PMid:8982370

20. Cupisti K, Wolf A, Raffel A, Schott M, Miersch D, Yang Q, et al Long-term clinical and biochemical follow-up in medullary thyroid carcinoma: A single institution's experience over 20 years. Ann Surg. 2007;246(5):815-21. https://doi.org/10.1097/ sla.0b013e31813e66b9

PMid: 17968174

21. Avaliable from: https://www.endocrineny.com/thyroid/bethesdaclassification.php. 